\title{
Use of a Clinical Audit System in Implementing Surviving Sepsis Campaign Guidelines in Patients With Peritonitis
}

\author{
Ramya C. Valiveru $^{1}$, Anusha Cherian ${ }^{2}$, Krishnamachari Srinivasan ${ }^{1}$, Nanda K. Maroju ${ }^{1}$ \\ 1. Surgery, Jawaharlal Institute of Postgraduate Medical Education and Research, Puducherry, IND 2. Anaesthesiology \\ and Critical Care, Jawaharlal Institute of Postgraduate Medical Education and Research, Puducherry, IND
}

Corresponding author: Anusha Cherian, anushacherian@gmail.com

\section{Abstract \\ Background}

Sepsis is the predominant cause of morbidity and mortality in patients with peritonitis. "Surviving Sepsis Campaign" (SSC) is an international effort in reducing mortality based on evidence-based guidelines. This study aims to assess the impact of audit-based feedback in a Plan-Do-Study-Act (PDSA) format on improving the implementation of the SSC guidelines in patients with generalized peritonitis at our center.

\section{Methods}

This prospective observational study was conducted in four audit cycles in PDSA format. Multi-departmental inputs were taken to suggest modifications in practice. A questionnaire-based analysis of reasons for noncompliance was performed to find out the opinions and reasons for non-compliance. Morbidity, mortality, and the length of ICU and hospital stay among these patients were also analyzed.

\section{Results}

Baseline compliance with intravenous (IV) bolus administration, central venous pressure (CVP)-guided fluids, and inotropes support when indicated were $100 \%$. Over the course of the three audit cycles, statistically significant improvement in compliance was noted for obtaining blood cultures before antibiotics, antibiotic administration within three hours of presentation, and serum lactate measurement. Overall bundle compliance improved from $9.2 \%$ to $64.7 \%$ by the end of audit cycle III.

\section{Conclusions}

This study demonstrates that audit-based feedback is a dependable means of improving compliance with SSC guidelines. It brings about improvement by educating users, modifying their behavior through feedback, and enhances process improvement by identifying and correcting systemic deficiencies in the organization.

Review began 06/16/2021 Review ended 06/19/2021 Published 06/27/2021

๑) Copyright 2021

Valiveru et al. This is an open access article distributed under the terms of the Creative Commons Attribution License CC-BY 4.0., which permits unrestricted use, distribution, and reproduction in any medium, provided the original author and source are credited.
Categories: Emergency Medicine, General Surgery, Quality Improvement

Keywords: surviving sepsis guidelines, audit, compliance, process improvement, peritonitis

\section{Introduction}

Sepsis is the predominant cause of mortality and morbidity in patients with peritonitis [1-4]. "Surviving Sepsis Campaign" (SSC) guidelines are a set of evidence-based recommendations, tailored for bedside implementation that was proposed in 2004 to combat sepsis. These were popularized as time stipulated bundles for resuscitation and maintenance [5]. Their implementation has proven to decrease mortality in severe sepsis and septic shock by many studies worldwide [6-8]. However, various studies have identified poor compliance with these guidelines among practitioners [9].

Lack of awareness of guidelines, disagreement with some components, lack of time, resources, motivation, impracticality of recommendations, and inertia toward adoption and adherence are some of the factors identified as reasons for poor compliance with the guidelines. Several methods have been attempted as means of improving compliance, including incorporating sepsis screen protocols into triage systems, creating sepsis carts and checklists, making pre-packed sepsis kits available in the emergency room, organizing multi-disciplinary sepsis response teams, and implementing sepsis stewardship programs [1011].

The role of clinical audit systems in implementation is well-established and is based on positive reinforcement through feedback. This study was conducted to analyze the role of a clinical audit system in implementing Surviving Sepsis Campaign guidelines in patients with generalized peritonitis. 


\section{Cureus}

\section{Materials And Methods}

This prospective observational study was conducted in the emergency department (ED) of a tertiary referral hospital over 21 months. All adult patients (> 18 years) presenting to the ED with generalized peritonitis during the study period were included. Patients with known rheumatic heart disease, congestive cardiac failure, and patients who received treatment prior to reaching the hospital were excluded. The Institutional Review Board approved this study, and all patients gave written informed consent to participate in the study.

The audit was performed in three cycles following a period of baseline data collection. The duration of the cycles was as follows: pre-audit cycle, October to March; audit cycle I, August to December; audit cycle II, January to April; and audit cycle III, May to July (Figure 1).

\section{Baseline data Collection User Initiation}

\section{Data driven meetings Direct User Interaction}

\section{Organisational redesign Wide publicity Intensive compliance reporting}

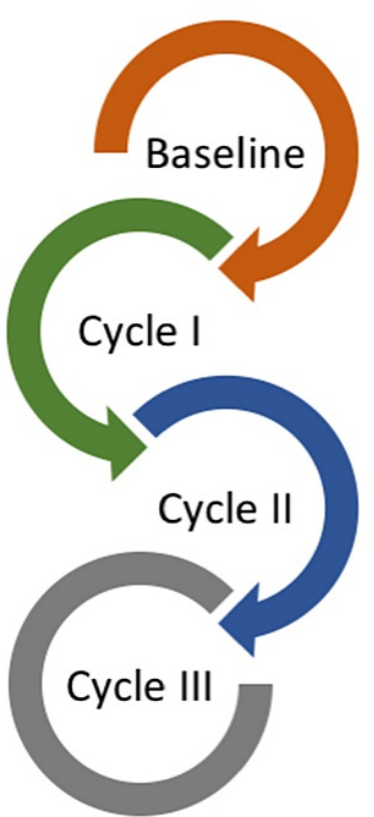

FIGURE 1: Audit cycle design

Auditing for compliance with components of the SSC resuscitation bundle was initiated as soon as the diagnosis of generalized peritonitis was established. The diagnosis of generalized peritonitis was made based on clinical features, radiographic or sonographic pneumoperitoneum, or sonography-guided aspirate of free bowel content, bile, or pus from the peritoneal cavity.

The guidelines followed during the study period are depicted in Figure 2. The first six steps of the guidelines were audited, including measuring serum lactate, blood cultures before antibiotics, antibiotics within three hours, initial fluid bolus, central venous pressure (CVP)-guided fluids when indicated, and inotropes when indicated. The seventh step of central venous oxygen saturation measurement was not included as a requirement owing to logistic reasons. 


\section{Cureus}

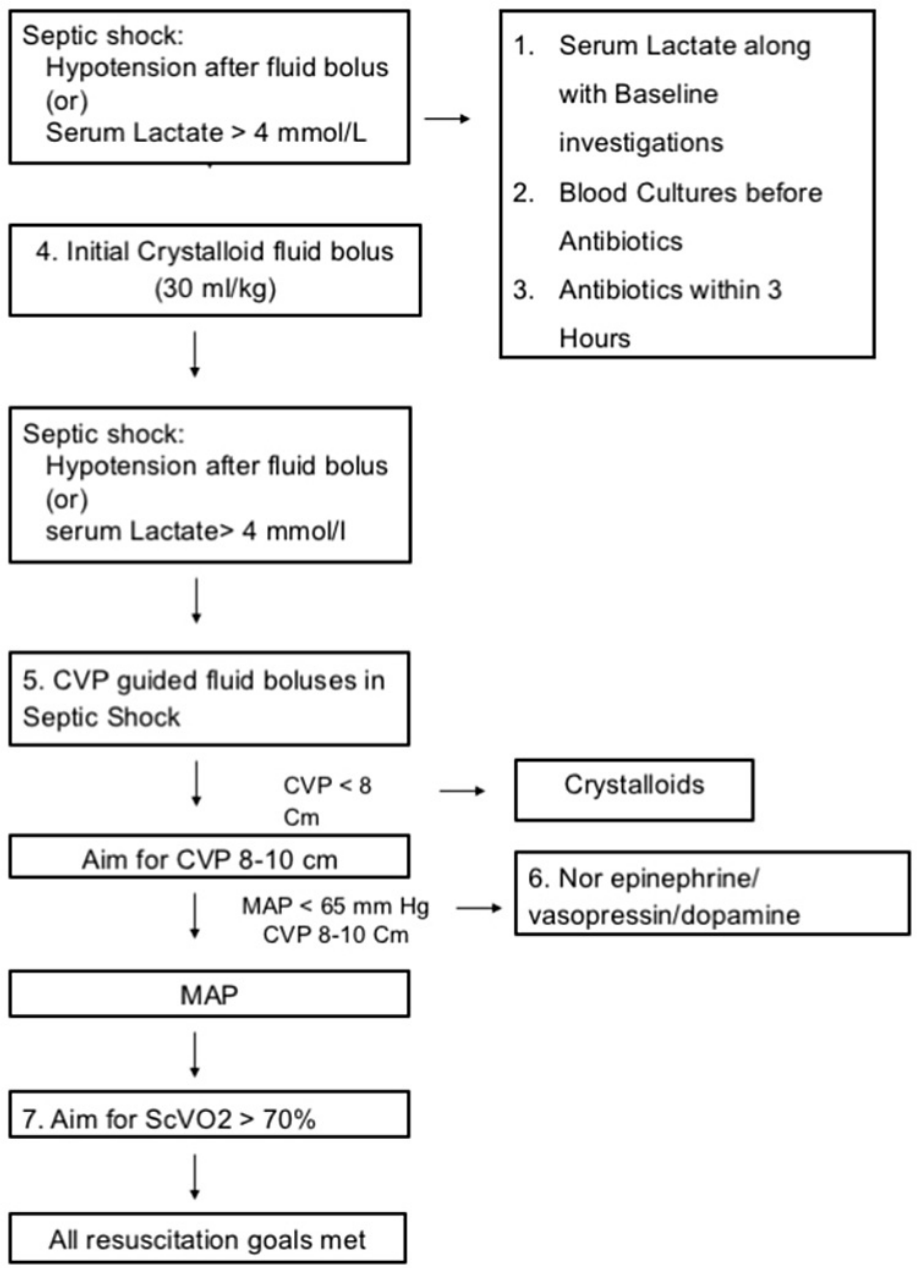

FIGURE 2: Components of the sepsis bundle

Compliance data were recorded in four cycles, and each cycle concluded with analysis and feedback. Multidepartmental inputs were sought for process change. The methods for process improvement were implemented as follows:

\section{Pre-audit}

Parameters were recorded to study the baseline compliance with the components of the guidelines and identify the areas of poor compliance and the probable causes for the same were presented in this meeting.

\section{Audit I}

The SSC coordinator initiated one-to-one communication with individual healthcare professionals regarding audit findings and provided feedback regarding day-to-day compliance.

\section{Audit II}

The consultant in charge of the audit sent audit feedback mailed to each consultant and resident. Colorcoded checklists were added in the ED to promote compliance with SSC guidelines.

\section{Audit III}

Compliance graphs and posters displaying the surviving sepsis resuscitation bundle were put up at all stations. Short group meetings were held with all the team members. 
Baseline data collected included patient demographics, date and time of admission, serum lactate, mean arterial pressures (MAP) at presentation, need for inotropes, and the Physiological and Operative Severity Score for the enUmeration of Mortality and Morbidity (POSSUM). Outcome variables were compliance with SSC guidelines, mortality rate, length of ICU stay, and length of hospital stay.

Compliance with each component of the SSC resuscitation bundle and the entire bundle was calculated. A questionnaire-based analysis of reasons for non-compliance was performed.

\section{Statistical analyses}

Descriptive statistics were used to compare compliance with each component of the resuscitation bundle in each cycle. Fischer's exact test and the chi-square test were used to compare categorical variables. One-way analysis of variance (ANOVA) was used to identify a statistically significant difference in the mean values between the two groups (mean \pm standard deviation). The Mann-Whitney U test was used for comparing non-parametric data. A two-sided $\mathrm{P}$ value of $<0.05$ was considered statistically significant. A statistical software package (InStat, Graphpad Software Inc, CA, USA) was used to perform statistical analyses.

\section{Results}

A total of 221 cases of generalized peritonitis were prospectively included in the four cycles of the audit, of which 65 patients were included for baseline data collection and 55, 50, and 51 patients were included in audit cycles I, II, and III, respectively. The demographic and baseline characteristics are presented in Table 1 .

\begin{tabular}{|c|c|c|c|c|}
\hline & Pre audit $(n=65)$ & Audit $(n=55)$ & Audit II ( $n=50)$ & Audit III ( $n=51)$ \\
\hline Age & $47.7 \pm 15.3$ & $48.2 \pm 15.0$ & $48.2 \pm 15.2$ & $44.1 \pm 15.7$ \\
\hline Male : Female & $5.5: 1$ & $4.5: 1$ & $5.6: 1$ & $5.3: 1$ \\
\hline Physiological score (POSSUM), mean \pm SD & $26.76 \pm 10.13$ & $27.58 \pm 9.18$ & $24.81 \pm 9.33$ & Not collected \\
\hline No. of shocked patients (MAP <65) at presentation & $39(60 \%)$ & $29(52.7 \%)$ & $21(42 \%)$ & $25(49 \%)$ \\
\hline ICU Stay, mean \pm SD & $3.2 \pm 2.9$ & $3.01 \pm 1.45$ & $2.28 \pm 1.69$ & $3.1 \pm 2.9$ \\
\hline Length of hospital stay, mean \pm SD & $11.9 \pm 11.3$ & $11.5 \pm 8.6$ & $9.1 \pm 5.7$ & $10.9 \pm 5.8$ \\
\hline Mortality, N(\%) & $21(32.3 \%)$ & $16(29 \%)$ & $12(24 \%)$ & $11(20 \%)$ \\
\hline
\end{tabular}

\section{TABLE 1: Patient characteristics}

* No statistically significant difference among the groups

Gastroduodenal perforation was the most common cause of peritonitis in all the audit cycles, followed by ileal-jejunal, appendicular, colonic, and gall bladder perforations in that order, and the least commonly encountered cause was a ruptured liver abscess.

Only $9.3 \%$ of patients presented within 24 hours of acute onset symptoms. About $15.2 \%$ of patients presented within 48 hours of the onset of symptoms, $30.2 \%$ patients within 72 hours. $45.5 \%$ of patients presented after a lag period (time between the onset of acute symptoms to arrival at the hospital) of more than 72 hours. A total of 39 (60\%), 29 (52.7\%), 21 (42\%), and 25 (49.01\%) patients in pre-audit, audit cycle I, audit cycle II, and audit cycle III, respectively, were in shock (mean arterial pressure (MAP) $<65 \mathrm{~mm} \mathrm{Hg}$ ) at presentation. The mean POSSUM physiological score at presentation was 26.7 in pre-audit, 27.5 in audit I, and 24.8 in audit II. The score could not be calculated in audit III due to certain logistic reasons. The POSSUM scores at admission in pre-audit, audit I, and audit II were comparable.

\section{Compliance with components of the sepsis bundle}

There was $100 \%$ compliance with respect to fluid bolus administration, CVP-guided fluid administration, and inotrope use at baseline.

Serum lactate was not performed in pre-audit, audit I, and audit II due to the lack of availability of the lactate measurement kits in the hospital. Serum lactate estimation was made available prior to the start of audit cycle III. Following its availability, serum lactate was performed in 40 patients (78.4\%) in audit cycle III. 


\section{Cureus}

Blood cultures before antibiotics were obtained only in nine patients (13.8\%) in pre-audit, 18 patients (32.72\%) in audit cycle I, 30 patients in audit cycle II (60\%), and 37 patients $(72.5 \%)$ in audit cycle III. The improvement was found to be statistically significant in audits I, II, and III when compared to pre-audit values.

Antibiotics were administered within three hours in only 30 patients (46.1\%) at baseline, 30 patients in audit cycle I (54.5\%), 40 patients in audit cycle II (80\%), and 46 patients in audit cycle III $(90.1 \%)$. The improvement observed in audit II and III was statistically significant when compared to the baseline values (Table 2).

\begin{tabular}{|c|c|c|c|c|}
\hline & $\begin{array}{l}\text { Pre-audit }(n=65) \\
(\%)\end{array}$ & $\begin{array}{l}\text { Audit cycle I ( } n=55) \\
(\%)\end{array}$ & $\begin{array}{l}\text { Audit cycle II ( } n=50) \\
(\%)\end{array}$ & $\begin{array}{l}\text { Audit cycle III ( } n=51) \\
(\%)\end{array}$ \\
\hline $\begin{array}{l}\text { Serum lactate measured at } \\
\text { admission }\end{array}$ & 0 & 0 & 0 & $40(78.4)$ \\
\hline Fluid bolus administration & $65(100)$ & $55(100)$ & $50(100)$ & $51(100)$ \\
\hline Blood cultures before antibiotics & $9(13.6)$ & $18(32.7)^{*}$ & $30(60)^{\star \star}$ & $37(72.54)^{\star \star}$ \\
\hline Antibiotics within $3 \mathrm{hrs}$. & $30(46.1)$ & $30(67.2)^{\star \star \star}$ & $40(80)^{\star \star \star \star}$ & $46(90.1)^{\star \star}$ \\
\hline CVP-guided fluids & $29 / 29(100)$ & 16/16 (100) & 8/8 (100) & $8 / 8(100)$ \\
\hline Inotropes when indicated & 17/17 (100) & 13/13 (100) & $5 / 5(100)$ & 4/4 (100) \\
\hline
\end{tabular}

\section{TABLE 2: Compliance with bundle components}

${ }^{\star} p=0.016,{ }^{\star \star} p<0.0001,{ }^{\star \star \star} p=0.46,{ }^{\star \star \star \star} p=0.0002$, when compared to pre-audit (baseline) values

CVP: central venous pressure

The overall bundle compliance increased from $9.2 \%$ in pre-audit to $23.6 \%, 54 \%$, and $64.7 \%$ through audit cycles I, II, and III (Table 3).

\begin{tabular}{|l|l|l|l|l|}
\hline $\begin{array}{l}\text { Total number of components } \\
\text { performed }\end{array}$ & $\begin{array}{l}\text { Pre-audit }(\mathbf{n}=65) \\
(\%)\end{array}$ & $\begin{array}{l}\text { Audit cycle I }(\mathbf{n}=55) \\
(\%)\end{array}$ & $\begin{array}{l}\text { Audit cycle II }(\mathbf{n}=50) \\
(\%)\end{array}$ & $\begin{array}{l}\text { Audit cycle III }(\mathbf{n}=51) \\
(\%)\end{array}$ \\
\hline $6 / 6$ & 0 & 0 & 0 & $33(64.7)$ \\
\hline $5 / 6$ & $6(9.2)$ & $13(23.6)$ & $27(54)$ & $3(5.8)$ \\
$4 / 6$ & $26(40)$ & $29(52.7)$ & $16(32)$ & $11(21.5)$ \\
$3 / 6$ & $33(50.7)$ & $13(23.6)$ & $7(14)$ & $4(7.8)$ \\
\hline
\end{tabular}

\section{TABLE 3: Total bundle compliance}

*Serum lactate was available in the hospital only during audit cycle III

\section{User perceptions}

Questionnaire-based analysis revealed that 48/50 (96\%) participants recognized the importance of performing all the bundle components. Among the responders, the main reasons for non-compliance were lack of time (12\%), tedious process (32\%), and lack of awareness (12\%). The most common reason for noncompliance for blood cultures before antibiotics was the lack of blood culture medium in the emergency department (66.6\%).

\section{Outcomes}

The overall mortality was found to be $32.3,29,24$, and $20 \%$ in pre-audit, audit cycle I, audit cycle II, and audit cycle III, respectively. The mean hospital stay was $11.95 \pm 11.36,11.5 \pm 8.64,9.16 \pm 5.77$, and $10.96 \pm$ 5.88 in pre-audit, and audit cycles I, II, and III, respectively. There was no significant difference in the audit 
cycles in terms of mortality or length of stay.

\section{Discussion}

A major concern in evidence-based medicine is the immense difficulty in translating research from the bench to the bedside irrespective of the level of evidence. This also becomes the main stumbling block in any process improvement program. Surviving Sepsis Guidelines incorporate a bundled care approach in the management of patients with sepsis. Implementing these guidelines is grossly inadequate in our center as it is in many other centers elsewhere. While the actual validity of these guidelines is constantly debated, and the guidelines are regularly revised, this study focused more on the implementation of the guidelines rather than the scientific validity or outcomes related to the guidelines. Accordingly, our observations apply to any process change initiative in healthcare.

This study was organized in the format of a Plan-Do-Study-Act (PDSA) cycle with multiple iterations. Defining the problem ensures that the process improvement team strategizes to achieve maximum success with maximum efficiency. Baseline data generation is an important component in defining the problem and brings out the strengths and weaknesses of the system and its users [12]. The baseline data obtained by us demonstrated our strengths and weaknesses. The data clearly demonstrated that fluid bolus administration, CVP-guided fluid administration, and inotrope use were applied in all cases, resulting in 100\% compliance among the components of the sepsis care bundles. The performance of serum lactate at presentation, obtaining blood cultures before antibiotic administration, administering antibiotics within three hours of presentation were infrequently adhered to. The total bundle compliance was a meager $9 \%$.

The three components that had 100\% compliance at baseline, namely, fluid bolus administration, CVPguided fluid administration, and inotrope use, are a part of standard resuscitation for patients presenting with hypovolemic shock. The data suggest that patients with peritonitis were managed as patients in hypovolemic shock, and therefore the components of the guidelines concerning hypovolemic shock were completely adhered to. This data identifies the strengths of the system in having a team that could reliably provide volume resuscitation [13]. The baseline data also demonstrate that specific guidelines for patients in sepsis were not adhered to. The message was to concentrate on training the staff and residents in recognizing the shock in peritonitis as septic rather than hypovolemic shock to the implementing team.

The interventions in audit cycle I aimed at sensitizing residents about the Surviving Sepsis Guidelines. Printed proformas were included at workstations of residents, and one-on-one interaction was conducted to emphasize the need to recognize septic shock and trigger the bundled approach to its management. At the end of this cycle, the total bundle compliance increased from $9 \%$ to $23 \%$. A significant improvement was seen both in the number of cases for which blood cultures were sent before antibiotics as well as in the rate of on-time antibiotic administration.

The improvement seen in audit cycle 1 was owing to the action taken by trainees who were already convinced and for the components that did not require much additional effort. In process improvement parlance, this is referred to as reaching for the "low-hanging fruit" [14]. Aiming for easy tasks at the beginning of any process improvement program achieves the twin objectives of boosting process improvement morale as well as winning over easy converts. It might make the task of further process improvement easier by recruiting more campaigners in the form of early converts.

Audit cycle II worked at consolidating the gains achieved over the first cycle, as well as becoming more aggressive in campaigning for compliance with the guidelines. This was done by giving individual user feedback with respect to their compliance. The proformas available at the acute surgery workstations were modified into checklists. Trainees were also encouraged to include the checklists as a part of their patient treatment records [15].

At the end of cycle II, total bundle compliance increased from $23 \%$ to $54 \%$. The improvement was seen in the number of cases for which blood cultures were sent before antibiotics, as well as in the rate of on-time antibiotic administration, which almost doubled from that seen in audit cycle I. This cycle also provided feedback in the form of systemic lacunae in the ED, which prevented full compliance with the guidelines.

An aggressive campaign directed by a person of responsibility or power can serve to increase compliance. However, this reaches a plateau limited by the conditions in the environment, as in the availability or limitations of material resources [16]. Unless the process improvement incorporates systemic changes, compliance is unlikely to improve. Cycle II brought out the systemic deficiencies to the fore, including poor inventory management, and an unconducive environment.

Prior to the start of audit cycle III, major changes in the ED in terms of streamlining the admission process, dedicated earmarking area for surgical admission, ownership of ED admission, and enhancement of nursing and physician presence were carried out at the Institutional level. Along with these changes, the process improvement team conducted small group meetings that included residents and nursing staff to reemphasize the need for following the guidelines. 
Serum lactate estimation was made available for the first time in the ED during this cycle. Total bundle compliance was $64 \%$, meaning that from a baseline level where less than one-tenth of the users complied, now more than two-thirds of users were complying with the guidelines. Ninety percent (90\%) of patients received on-time antibiotics while blood cultures at admission were sent for $72 \%$ of patients. Systemic changes not only made it easier for users to comply with proposed guidelines, but they also convince the user regarding the seriousness of the organization in improving the process. In other words, it is easier to ensure user buy-in of process change if the organization also takes concrete measures towards that process change.

Small group meetings at this stage allowed for a two-way discussion between the process improvement team and the users to understand each other's viewpoints as well as agreeing upon the next course of action towards realizing shared objectives. This phase was associated with shifting the ownership of implementation from the implementation team to the clinical team.

This study did not demonstrate any improvement in the broad outcomes of mortality and length of stay. The possible reasons for this are two-fold. First, this study was not designed to detect the difference in outcomes. Second, the numbers needed to detect a change in outcomes following the implementation of SSC guidelines far exceed the patient numbers in each audit cycle.

The audit was conducted without undue pressure to modify practice, thus reducing the impact of the "Hawthorne effect" [17]. However, this study had several limitations. The hospital was not equipped with point of care lactate estimation, thus limiting the possibility of achieving full compliance initially. Outcome analysis was not performed in a reliable manner. The process improvement team was an internal team and lacked prior expertise in conducting large-scale process improvement programs.

\section{Conclusions}

A clinical audit system is a dependable tool to implement surviving sepsis campaign guidelines. It brings about improvement by educating users, behavioral modification by providing feedback, and enhances process improvement by identifying and correcting systemic deficiencies in the organization. This study reinforces the importance of a multimodal approach in quality improvement and implementation.

\section{Additional Information}

\section{Disclosures}

Human subjects: Consent was obtained or waived by all participants in this study. Institute Ethics Committee (Human Studies), JIPMER, Puducherry issued approval IEC/SC/2012/5/231. Animal subjects: All authors have confirmed that this study did not involve animal subjects or tissue. Conflicts of interest: In compliance with the ICMJE uniform disclosure form, all authors declare the following: Payment/services info: All authors have declared that no financial support was received from any organization for the submitted work. Financial relationships: All authors have declared that they have no financial relationships at present or within the previous three years with any organizations that might have an interest in the submitted work. Other relationships: All authors have declared that there are no other relationships or activities that could appear to have influenced the submitted work.

\section{References}

1. Afridi SP, Malik F, Ur-Rahman S, Shamim S, Samo KA: Spectrum of perforation peritonitis in Pakistan: 300 cases Eastern experience. World J Emerg Surg. 2008, 3:31. 10.1186/1749-7922-3-31

2. Noguiera C, Silva AS, Santos JN, Silva AG, Ferreira J, Matos E, Vilaça H: Perforated peptic ulcer: main factors of morbidity and mortality. World J Surg. 2003, 27:782-7. 10.1007/s00268-003-6645-0

3. Søreide K, Thorsen K, Søreide JA: Strategies to improve the outcome of emergency surgery for perforated peptic ulcer. Br J Surg. 2014, 101:e51-64. 10.1002/bjs.9368

4. Bali RS, Verma S, Agarwal PN, Singh R, Talwar N: Perforation peritonitis and the developing world. ISRN Surg. 2014, 2014:105492. 10.1155/2014/105492

5. Marshall JC, Dellinger RP, Levy M: The Surviving Sepsis Campaign: a history and a perspective . Surg Infect (Larchmt). 2010, 11:275-81. 10.1089/sur.2010.024

6. Li ZQ, Xi XM, Luo X, Li J, Jiang L: Implementing surviving sepsis campaign bundles in China: a prospective cohort study. Chin Med J (Engl. 2013, 126:1819-1825.

7. Wang Z, Xiong Y, Schorr C, Dellinger RP: Impact of sepsis bundle strategy on outcomes of patients suffering from severe sepsis and septic shock in China. J Emerg Med. 2013, 44:735-41.

10.1016/j.jemermed.2012.07.084

8. Castellanos-Ortega A, Suberviola B, García-Astudillo LA, Holanda MS, Ortiz F, Llorca J, Delgado-Rodríguez M: Impact of the Surviving Sepsis Campaign protocols on hospital length of stay and mortality in septic shock patients: results of a three-year follow-up quasi-experimental study. Crit Care Med. 2010, 38:1036-43. 10.1097/CCM.0b013e3181d455b6

9. Zambon M, Ceola M, Almeida-de-Castro R, Gullo A, Vincent JL: Implementation of the Surviving Sepsis Campaign guidelines for severe sepsis and septic shock: we could go faster. J Crit Care. 2008, 23:455-60. 10.1016/j.jcrc.2007.08.003

10. Kang MJ, Shin TG, Jo IJ, et al.: Factors influencing compliance with early resuscitation bundle in the 


\section{Cureus}

management of severe sepsis and septic shock. Shock. 2012, 38:474-9. 10.1097/SHK.0b013e31826eea2b

11. Kakebeeke D, Vis A, de Deckere ER, Sandel MH, de Groot B: Lack of clinically evident signs of organ failure affects ED treatment of patients with severe sepsis. Int J Emerg Med. 2013, 6:4. 10.1186/1865-1380-6-4

12. Gerber K: Surviving sepsis: a trust-wide approach. A multi-disciplinary team approach to implementing evidence-based guidelines. Nurs Crit Care. 2010, 15:141-51. 10.1111/j.1478-5153.2009.00378.x

13. Paltridge D, Dent AW, Weiland TJ: Australasian emergency physicians: a learning and educational needs analysis. Part two: confidence of FACEM for tasks and skills. Emerg Med Australas. 2008, 20:58-65. 10.1111/j.1742-6723.2007.01037.x

14. Clack L, Kuster SP, Giger H, Giuliani F, Sax H: Low-hanging fruit for human factors design in infection prevention-still too high to reach?. Am J Infect Control. 2014, 42:679-81. 10.1016/j.ajic.2014.03.002

15. Rezazadeh E, Hachesu PR, Rezapoor A, Alireza K: Evidence-based medicine: going beyond improving care provider viewpoints, using and challenges upcoming. J Evid Based Med. 2014, 7:26-31. 10.1111/jebm.12083

16. Lowson K, Jenks M, Filby A, Carr L, Campbell B, Powell J: Examining the implementation of NICE guidance: cross-sectional survey of the use of NICE interventional procedures guidance by NHS Trusts. Implement Sci. 2015, 10:93. 10.1186/s13012-015-0283-4

17. Yanes AF, McElroy LM, Abecassis ZA, Holl J, Woods D, Ladner DP: Observation for assessment of clinician performance: a narrative review. BMJ Qual Saf. 2016, 25:46-55. 10.1136/bmjqs-2015-004171 